\title{
The New WHO Growth Standards
}

\section{Comparison with Other Growth Charts}

\author{
Ekhard E. Ziegler Steven E. Nelson \\ Department of Pediatrics, University of lowa, lowa City, lowa, USA
}

\section{Key Words}

WHO growth standards $\cdot$ Growth charts

\begin{abstract}
The new World Health Organization (WHO) growth standards were created with the intention of producing globally applicable growth standards that describe the growth of children as it occurs under optimal nutritional conditions and in the absence of external constraints. In other words, it represents childhood growth as it should be. Children in six countries living in favorable circumstances provided data for the new charts. In addition, data for some subjects at the extremes of weight for height were excluded. The new WHO growth standards, not unexpectedly, differ from other growth charts in a number of ways. Because of the many differences, only a few general statements can be made. (1) During the first 6 months of life, WHO weight and length at all percentiles are larger than weight and length by any other chart. (2) During the second 6 months of life, and continuing through the 2 nd year of life, WHO weight (but not length) is lower than weight by other charts. (3) Between 2 and 5 years of age, $\mathrm{WHO}$ weight tends to be at the lower end of the spectrum, especially at the lower percentiles, whereas EuroGrowth occupies the top end for weight at all percentiles. (4) Functional assessment shows that the WHO charts identify fewer 1- to 2-year-olds as underweight and more 2- to 5year-olds as overweight than other charts.
\end{abstract}

Copyright $\odot 2008$ Nestec Ltd., Vevey/S. Karger AG, Basel
(C) 2008 Nestec Ltd., Vevey/S. Karger AG, Basel 0517-8606/07/0653-0109\$23.50/0

Fax +4161306 1234 E-Mail karger@karger.ch www.karger.com
Accessible online at:

www.karger.com/ane
Growth charts are important tools in the assessment of growth and nutritional status of individual children (clinical use) and in the assessment of groups of children (epidemiologic use). Growth charts are perceived to represent the normal growth of children and therefore carry normative authority. Although many countries have national growth charts, a great many do not. For use in such countries, and also for facilitation of international comparisons, there is a need for universal growth charts. To meet this need, the World Health Organization (WHO) in 1978 adopted growth charts which had been constructed by the National Center for Health Statistics (NCHS) of the United States of America [1]. The charts became known as the NCHS/WHO growth charts.

But before long it became apparent that the NCHS/ WHO charts were not representing the growth of infants very faithfully. In comparison with the weight of breastfed infants in Europe and the United States [2], the weight for age of the NCHS/WHO charts was shown to be unduly low in the first 6 months of life, whereas in the second 6 months NCHS weight tended to be higher than that of breastfed infants [3]. These shortcomings were thought to derive mainly from two features of the underlying growth study. First, anthropometric data during the first year of life were spaced too widely (every 3 months). Second, few of the infants were fully breastfed, and of those who were breastfed many were breastfed for only a short period. It was also considered disadvanta-

Ekhard E. Ziegler, MD

Department of Pediatrics

University of Iowa, A 136 MTF, 2501 Crosspark Rd.

Iowa City, IA 52242 (USA)

Tel. +1 319335 4570, Fax +1 319335 4856, E-Mail ekhard-ziegler@uiowa.edu 
geous that the subjects lived in a single geographic area of the United States. A WHO-convened Expert Committee determined that the NCHS/WHO charts were inappropriate and inadequate and recommended that new charts for global use be created [3]. A Multicentre Growth Reference Study was initiated and data for new internationally representative charts for children from birth to 5 years were gathered between 1997 and 2003. The new WHO Growth Standards for global use were released in 2006 [4].

Traditionally, growth charts have described the growth of children who are considered to be normal and healthy and who are living in a defined geographic area, whether it be a single country, as in the case of the NCHS charts [1], or a group of countries, as in the case of the EuroGrowth charts [5]. Such charts are referred to as growth references. The new WHO growth charts depart from this tradition and do not simply describe the growth of actual normal children. Rather, they aspire to represent the optimal growth of children. Operationally this was defined as the growth of children living under conditions that pose no environmental restraint on growth. In the case of infants and toddlers, it further meant being fed according to $\mathrm{WHO}$ recommendations. The WHO charts are therefore classified as 'standards', in contrast to 'references' that describe the growth of all children, including those who are not living under favorable conditions. The WHO standards also sought to be broadly internationally representative.

While the new WHO charts were being developed, two new charts were released. The Euro-Growth charts, resulting from a Europe-wide multicenter growth study [5], were released in the year 2000. Also in 2000, the Centers for Disease Control and Prevention (CDC) released new US-based growth charts [6, 7], which were the result of a revision of the NCHS charts that was undertaken with the intent to correct the shortcomings of the latter.

The new WHO growth standards are presented here in comparison to the NCHS/WHO charts, which they replaced, and also the Euro-Growth and CDC charts. We show in what ways the WHO charts differ from the other charts and also how the other charts differ from each other. Although many of the differences are too small to be of any impact, some of the differences are quite large and are likely to have functional consequences.

\section{The New WHO Growth Standards}

A Multicentre Growth Reference Study was carried out between 1997 and 2003 with the objective of describing the growth of children living under conditions that posed no constraints on growth. Because the intention was to create an internationally representative standard, data were gathered at 6 sites in 6 countries (Brazil, Ghana, India, Norway, Oman, USA). The study consisted of two parts, a longitudinal study in which subjects were followed from birth to 2 years of age, and a cross-sectional study of children between 1.5 and 5 years of age. It is worth noting that, as all other charts, the WHO charts from birth to 2 years represent recumbent length and only from 2 years on do they represent standing height.

\section{Longitudinal Study}

Of 1,737 subjects enrolled in the longitudinal study, 882 completed the study and only their data were used. Infants born at term were included regardless of birth weight, so that the sample included $2.3 \%$ of infants with a birth weight of $<2,500 \mathrm{~g}$. Subjects were fed according to WHO recommendations, meaning that they were breastfed for the first 12 months of life with complementary foods introduced only after 6 months of age. Their mothers did not smoke cigarettes. Application of the strict eligibility criteria in some countries led to the exclusion of the majority of subjects in the respective age brackets, whereas in other countries eligibility criteria excluded only a small portion of subjects. It meant also that subjects generally came from the more privileged strata of societies.

\section{Cross-Sectional Study}

Subjects were recruited from the same demographic strata as subjects in the longitudinal study. The 6,669 subjects aged 18-71 months were each measured on one occasion. Because of the adverse health effects of being overweight, data for subjects with weight for height $>2$ $\mathrm{SD}$ were excluded from the cross-sectional sample. In this way data for $1.4 \%$ of boys and $1.1 \%$ of girls were excluded.

\section{Other Growth Charts}

The NCHS 1977 Growth Curves [1] from birth to 3 years were based on longitudinal data collected by the Fels Institute between 1929 and 1975 [8]. The subjects 
were predominantly white and were living in a narrow geographic area of the United States. Although the majority of subjects was breastfed as infants, some subjects were breastfed for short periods only, and a substantial minority was not breastfed at all. Measurements of weight and length were made every 3 months during the first year of life. For ages 2-18 years NCHS relied on nationally representative cross-sectional data from National Health Examination Surveys.

The CDC 2000 Charts [6, 7] represent a modification of the NCHS charts. From birth to 3 years, the original longitudinal data were replaced by cross-sectional data from 5 nationally representative surveys conducted in the US between 1963 and 1994. Because of a paucity of data for infants, additional data for length (but not weight) from government-sponsored health clinics (Women, Infants and Children) were used. In all, weight data were available only for fewer than 300 subjects/ month (boys and girls) between 3 and 12 months (no weight data for months 1 and 2). Length data were available from large numbers of subjects for months $1-5$, but for fewer than 300/month between 6 and 12 months. Because of the limited number of subjects, confidence in the accuracy of the outer percentiles is low. No information about the nutrition of infants is available. Weight data for children $>6$ years obtained between 1988 and 1994 were excluded because of the high prevalence in that cohort of children with 'unhealthy' (i.e., high) weights.

Euro-Growth 2000 Charts [5]. Data for the EuroGrowth charts were gathered at 22 sites in 11 European countries. Subjects born between 1990 and 1993 were followed longitudinally from birth to 5 years. Of 2,245 subjects enrolled, 1,746 completed the study to age 12 months, 1,205 to age 24 months and 1,071 to age 3 years. Subjects were born after 37 or more weeks of gestation with a birth weight of $>2,500 \mathrm{~g}$. A minority of subjects were fed according to WHO recommendations, i.e., breast-feeding for 1 year, complementary foods only after 6 months.

\section{Functional Assessment}

The functional impact of the differences between charts was assessed with the help of hypothetical samples. These samples had normally distributed weight and length/height and were centered on the 50th percentile of the NCHS charts.

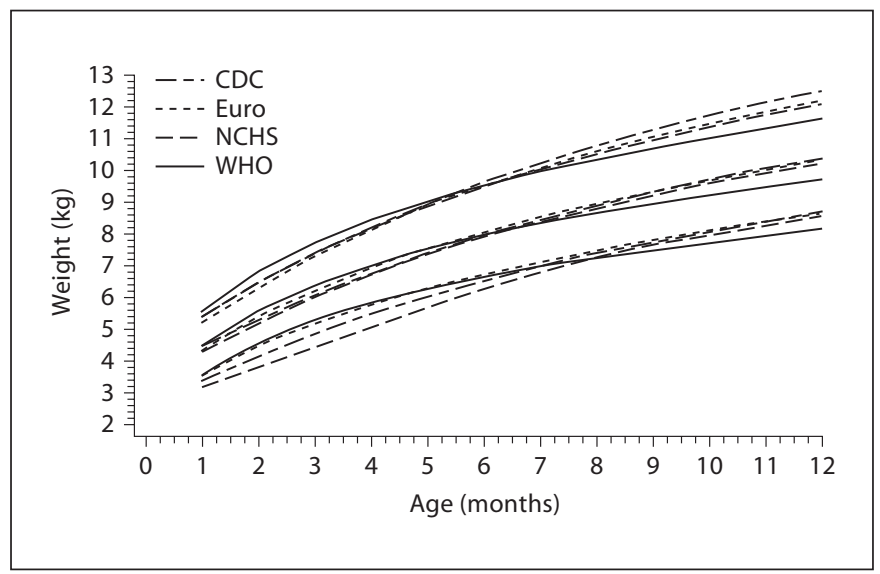

Fig. 1. Weight for age of male infants aged 1-12 months. The 5th, 50th and 95th percentiles for WHO in comparison with Euro, $\mathrm{NCHS}$ and CDC charts are shown.

\section{The WHO Standards in Comparison}

\section{First Year of Life}

Figure 1 shows the 5th, 50th and 95th percentiles for weight for age for boys during the first year of life of the new WHO growth standard in comparison with the three other charts. (The relations among charts are in every case similar for girls and charts for girls are not shown separately.) It is evident that during the first 6 months WHO weight tends to be greater than the weight of other charts at all percentiles. During the second 6 months of life the reverse is true, with WHO weight being progressively lower than the weight on other charts. The magnitude of the differences is not the same for all percentiles. During the first 6 months differences are particularly large for the 5th percentile, whereas during the second semester differences are rather homogeneous. To facilitate comparison, figure 2 shows the 50th percentile of the other charts expressed as SD units (z scores) of the WHO standards. During the first 6 months the differences reach $0.3-0.6 \mathrm{SD}$ units. After 6 months the differences reach about 0.5 SD units at 12 months, albeit in the opposite direction.

For length (fig. 3), differences between the WHO standards and other charts are less dramatic than for weight. The spread between outer percentiles tends to be narrower for the WHO standards than for the other charts. As figure 4 illustrates, the differences at the 50th percentile tend to be less than $0.3 \mathrm{SD}$ units. Contrary to CDC weight, CDC length differs markedly from NCHS length and Euro-Growth length; an explanation for this discrepancy is not available. 


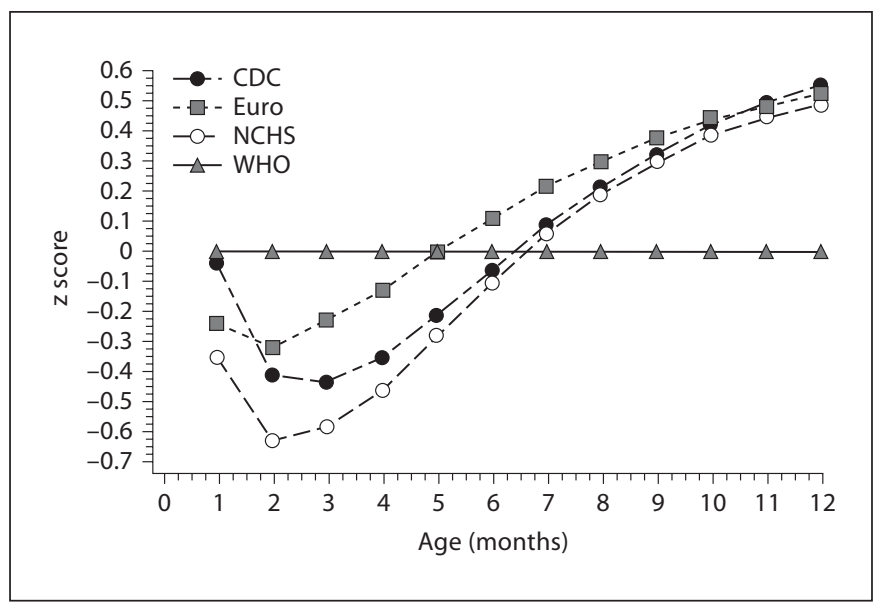

Fig. 2. 50th percentile of weight for age (male and female infants) of Euro, CDC and NCHS charts expressed as WHO charts z scores.

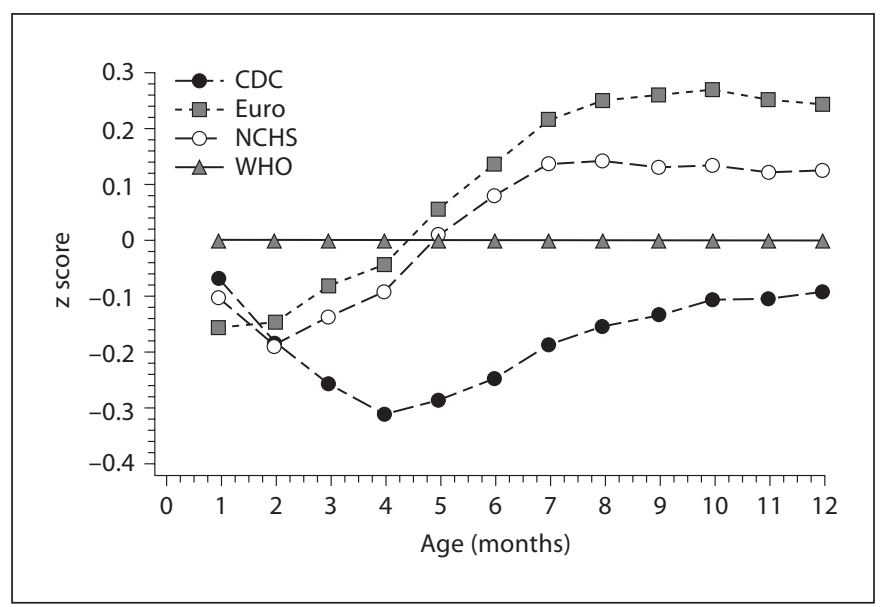

Fig. 4. 50th percentile of length for age (male and female infants) of Euro, CDC and NCHS expressed as WHO z scores.

To gain some perspective on the sources of differences between charts, published $[2,9,10]$ and unpublished growth data for breastfed and formula-fed infants were used. Comparison with these data (not shown) leads to the conclusion that during the first 6 months WHO and Euro-Growth represent the growth of infants more faithfully than NCHS and CDC. Differences in weight between breastfed and formula-fed infants are much smaller than differences between WHO, on the

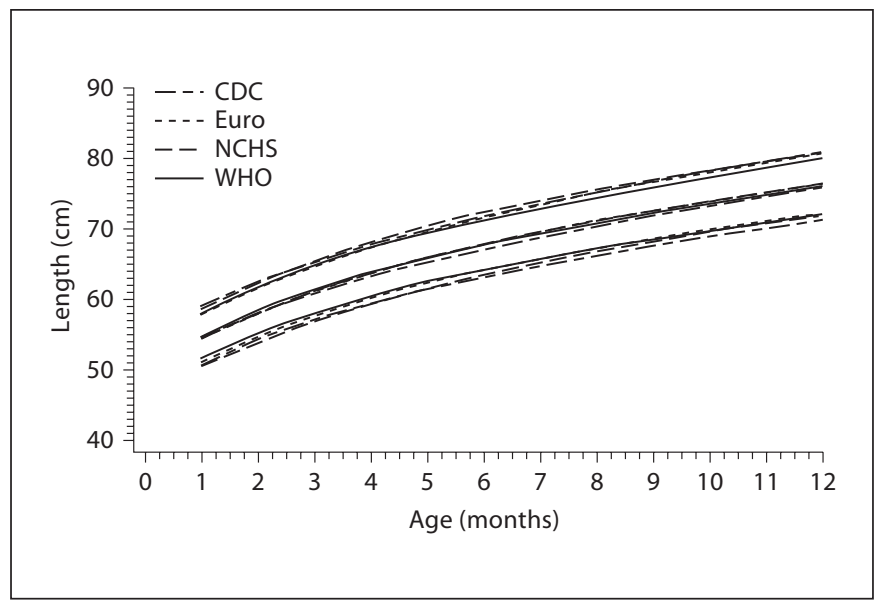

Fig. 3. Length for age of male infants aged 1-12 months. The 5th, 50 th and 95th percentiles for WHO in comparison with Euro, NCHS and CDC charts are shown.

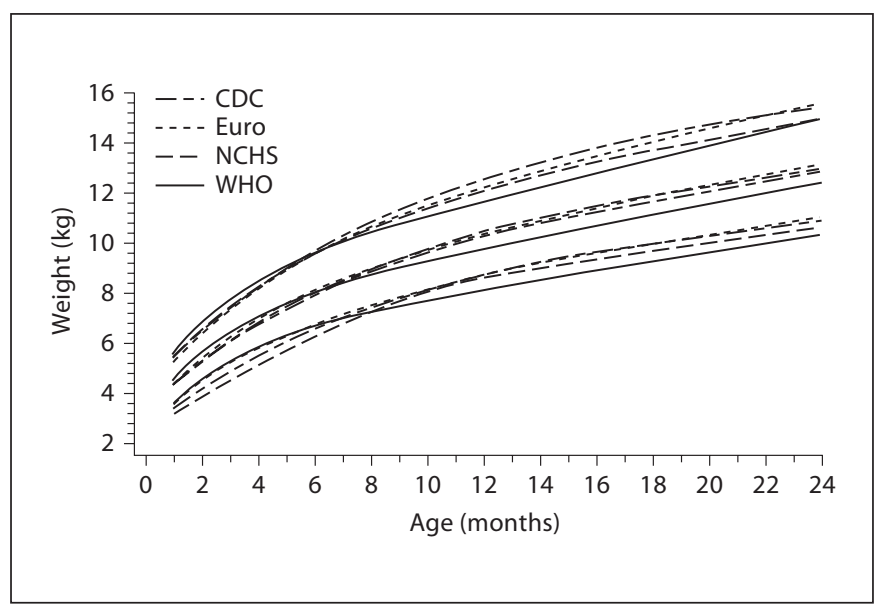

Fig. 5. Weight for age of male children aged 12-24 months. The 5th, 50th and 95th percentiles for WHO in comparison with Euro, NCHS and CDC charts are shown.

one hand, and NCHS and CDC charts, on the other. This leads to the further conclusion that the shortcomings of the NCHS charts and also the CDC charts in the first 6 months of life are not a reflection of differences in feeding regimens (i.e., breast vs. formula) but instead have their origins elsewhere, e.g. paucity of measurements and, probably, methods of curve fitting and smoothing. These comparisons also led to the conclusion that the CDC charts do not represent a substantial 


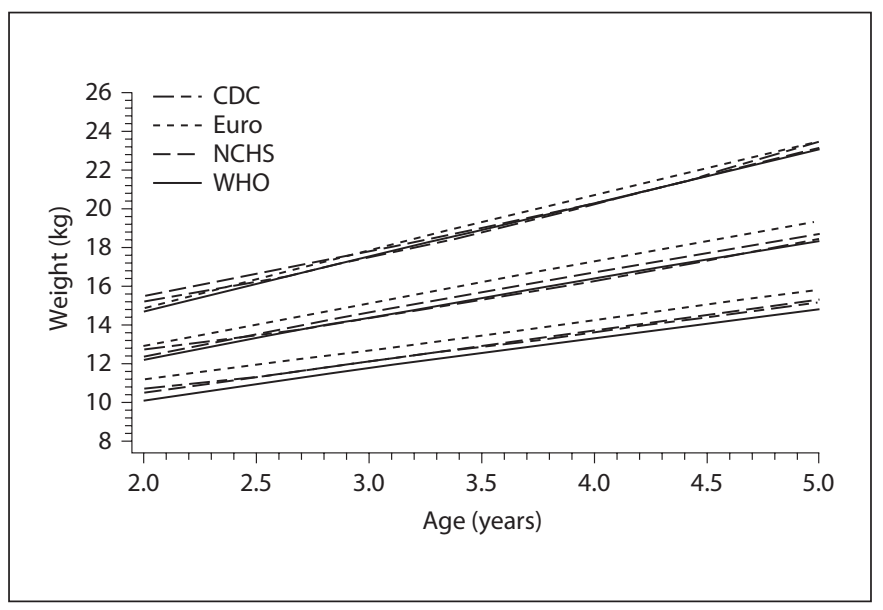

Fig. 6. Weight for age of male children aged 2-5 years. The 5 th, 50th and 95th percentiles for WHO in comparison with Euro, NCHS and CDC charts are shown.

improvement beyond the inadequate state of their predecessor, the NCHS charts. Neither NCHS nor CDC represent the growth of infants in the first 6 months very well.

\section{Second Year of Life}

Trends and differences that emerge during the second 6 months of the first year continue during the second year of life. As figure 5 shows, all weight percentiles of the WHO charts are lower than those of the other growth charts. Length percentiles (not shown) similarly continue on trajectories established between 6 and 12 months, with the WHO charts continuing to show a narrower spread between outer percentiles than other charts.

\section{Two-Five Years}

Between 2 and 5 years there are marked differences between charts in weight (fig. 6) and height (fig. 7). EuroGrowth weight (but not length) tends to be greater than weight of other charts by a considerable margin (0.4-0.6 SD units), for which an explanation is not readily available. WHO weight percentiles tend to occupy the lower end of the spectrum. This is explained, at least for the upper percentiles, by the exclusion of data of some of the heaviest children. The peculiar pattern of CDC weight between 2 and 3 years results from the linking of charts for infants with those for children.

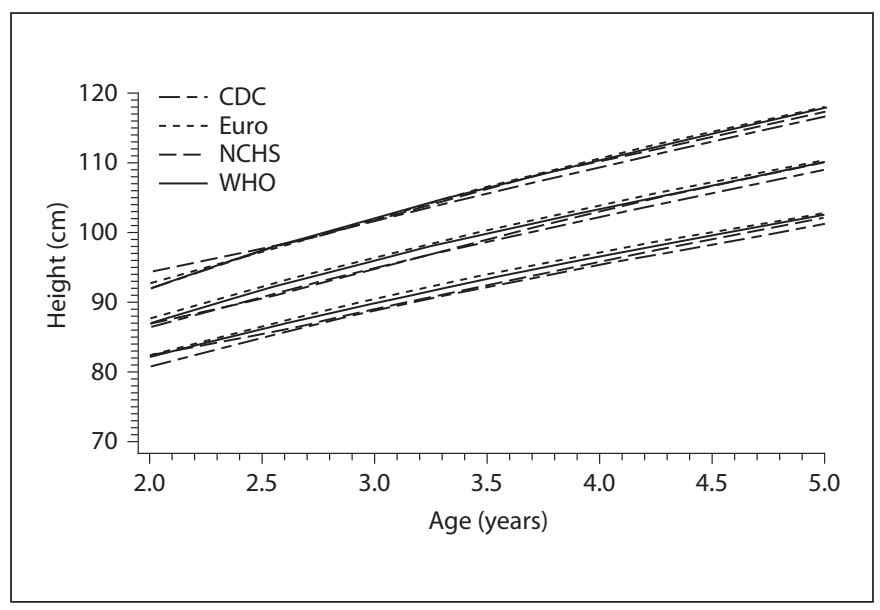

Fig. 7. Height for age of male children aged 2-5 years. The 5 th, 50th and 95th percentiles for WHO in comparison with Euro, NCHS and CDC charts are shown.

\section{Functional Assessment}

Figure 8 compares the percentages of normal children aged 1-2 years identified as being underweight (weight $<10$ th percentile). The proportion of normal children identified as being underweight is markedly lower with use of the WHO charts than with other charts, although differences among other charts are also substantial. Using the WHO charts, about $3 \%$ of children are identified as underweight, whereas using the NCHS charts about $8 \%$ are identified as underweight and, using the CDC and Euro curves, about $13-14 \%$ are identified as underweight. It is conceivable that the use of the new WHO charts could lead to delayed recognition of growth failure occurring among formula-fed infants/toddlers.

Another example is presented in figure 9. Among normal 2- to 5-year-old boys, the proportion identified as overweight (BMI >90th percentile) is about $16 \%$ with use of the WHO charts but is only $11 \%$ with the CDC charts and about $7 \%$ with the Euro-Growth charts.

These examples illustrate the sizable differences in proportions of children identified when the various charts are used for screening purposes. There are differences among all charts, but for some of the more important variables (fig. 8,9 ) the WHO charts are at the extreme of the spectrum. This is largely the intended effect of the prescriptive approach used by WHO. 


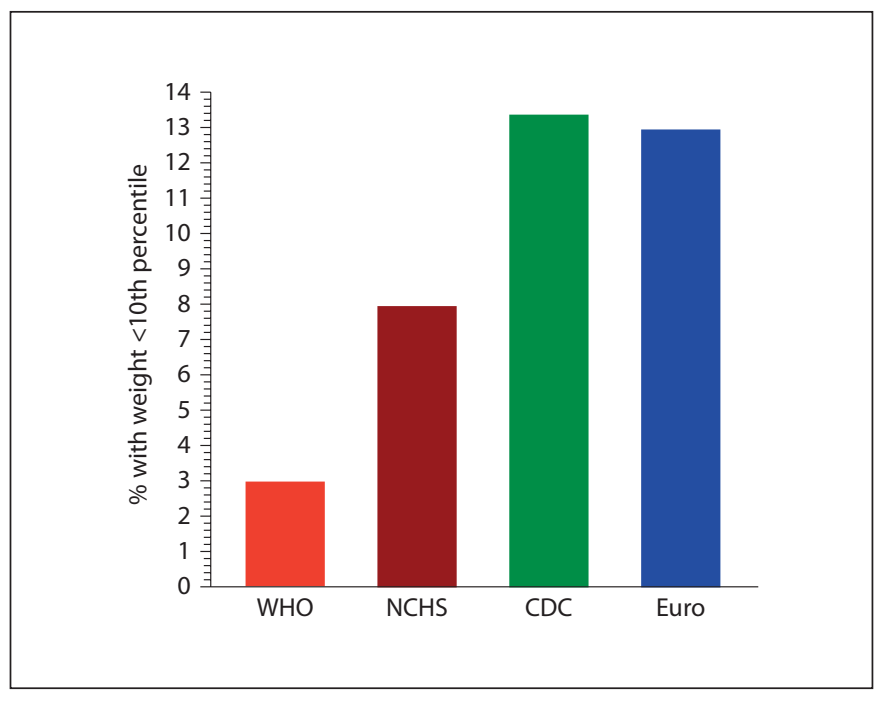

Fig. 8. Percentage of normal 1- to 2-year-old children identified as underweight using the WHO, NCHS, CDC or Euro growth charts.

\section{Comment}

The new WHO growth charts were created using a prescriptive approach and aspire to describe the growth of children as it proceeds in an optimal fashion and is unimpeded by external factors. The WHO growth charts differ in major ways from existing growth charts. Although there are many differences among existing growth charts, the new WHO charts set themselves apart by following a different pattern. This is largely the result of the prescriptive approach. Differences are far from uniform, changing in direction and varying in magnitude with age. For example, in the first 6 months, WHO weight is greater than the weight on any other chart, whereas in the second 6 months WHO weight is lower than the weight on any other chart. The differences in weight in the first 6 months are not fully explained by differences in feeding regimens and are in part the result of other factors, mostly of a technical nature.

For the use of charts in the management of individual children (clinical use) the differences between charts are probably of minor consequence. After all, the clinician usually evaluates growth on the basis of multiple measurements and tends to judge growth by the extent to which it proceeds in parallel to percentiles. Thus, for clinical use the position of percentiles is of less importance than the direction of percentiles. On the other hand, in the epidemiologic use of growth charts,

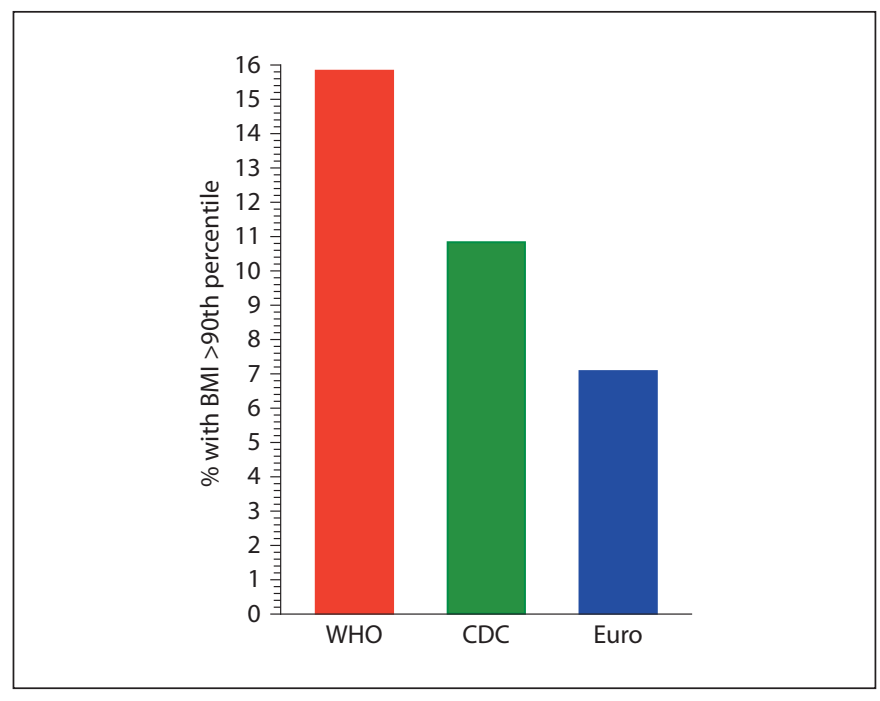

Fig. 9. Percentage of normal 2- to 5-year-old children identified as overweight using the WHO, CDC or Euro growth charts.

where the objective is to determine the prevalence of aberrant growth, the position of outer percentiles is of greatest interest. Here differences between growth charts may be of considerable impact. As the examples in figures 8 and 9 illustrate, the proportion of subjects identified by the various charts varies greatly. In the case of the WHO chart, this effect is intentional. The WHO chart wishes, for example, to identify more children as overweight than other charts, presumably because thereby the problem of childhood overweight would receive more attention. Therefore, adjusting traditional cutoffs would seem to defeat the intention of the WHO charts.

References

1 Hamill PV, Drizd TA, Johnson CL, Reed RB, Roche AF: NCHS growth curves for children birth-18 years. United States. Vital Health Stat 11 1977; 165:i-iv, 1-74.

-2 Dewey KG, Peerson JM, Brown KH, Krebs NF, Michaelsen KF, Persson LA, Salmenpera L, Whitehead RG, Yeung DL, WHO Working Group on Infant Growth: Growth of breast-fed infants deviates from current reference data: a pooled analysis of US, Canadian and European data sets. Pediatrics 1995;96:495-503.

-3 de Onis M, Habicht JP: Anthropometric reference data for international use: recommendations from a World Health Organization Expert Committee. Am J Clin Nutr 1996;64:650-658. 
-4 WHO Multicentre Growth Reference Study Group: WHO child growth standards based on length/height, weight and age. Acta Paediatr Suppl 2006;450:76-85.

5 Haschke F, van t'Hoff MA (eds): EuroGrowth. J Pediatr Gastroenterol Nutr 2000; 31(suppl 1):S1-S86.

6 Kuczmarski RJ, Ogden CL, Grummer-Strawn LM, Flegal KM, Guo SS, Wei R, Mei Z, Roche AF, Johnson CL: CDC growth charts: United States. Adv Data 2000;314:1-27.
7 Ogden CL, Kuczmarski RJ, Flegal KM, Mei Z, Guo S, Wei R, Grummer-Strawn LM, Curtin LR, Roche AF, Johnson CL: Centers for Disease Control and Prevention 2000 growth charts for the United Sates: improvements to the 1977 National Center for Health Statistics version. Pediatrics 2002;109:45-60.

8 Roche AF: Growth, Maturation and Body Composition: The Fels Longitudinal Study 1929-1991. Cambridge, Cambridge University Press, 1992.
9 Nelson SE, Rogers RR, Ziegler EE, Fomon SJ: Gain in weight and length during early infancy. Early Hum Dev 1989;19:223-239.

10 Haschke F, van t'Hoff MA; Euro-Growth Study Group: Euro-Growth references for breast-fed boys and girls: influence of breastfeeding and solids on growth until 36 months of age. J Pediatr Gastroenterol Nutr 2000; 31(suppl 1):S60-S71. 